\title{
TRATAMENTO DA REJEIÇÃO HUMORAL AGUDA EM RECEPTORES DE TRANSPLANTE RENAL
}

\author{
Treatment of the Acute Humoral Rejection in Kidney Allografts \\ Adriana Reginato Ribeiro', Roberto Herz Berdichevski', Daniel Melquíades da Silva', Marilda Mazzali², \\ Luiz Felipe dos Santos Gonçalves', Roberto Ceratti Manfro ${ }^{1}$
}

\begin{abstract}
RESUMO
Introdução: A rejeição humoral aguda (RHA) é uma complicação grave do transplante renal usualmente associada a mau prognóstico do enxerto. Recentemente tem sido proposta uma abordagem terapêutica com plasmaferese e imunoglobulinas intravenosas (IVIg). Objetivo: Relatar a efetividade do tratamento da RHA. Método: Durante o período de janeiro de 2002 a abril de 2008 foi feito diagnóstico de RHA em 15 pacientes que foram tratados com plasmaferese combinada com IVIg ou ATG/OKT3 em nossa instituição. Resultados: A reversão da RHA foi obtida em doze casos (80\%). A sobrevida de pacientes e enxertos em um ano foram respectivamente, $100 \%$ e $73 \%$. Um paciente tratado com sucesso desenvolveu sepse e teve sua imunossupressão descontinuada com conseqüente perda do enxerto. Conclusão: Conclui-se que essa abordagem terapêutica é eficiente e permite a sobrevida da maioria dos enxertos.
\end{abstract}

Descritores: Transplante de Rim, Rejeição de Enxerto, Plasmaferese, Imunoglobulinas, Soro Antilinfocitário, Muromonab-CD3.

\footnotetext{
Instituições:

1 Serviço de Nefrologia. Unidade de Transplante Renal. Hospital de Clinicas de Porto Alegre / RS

${ }^{2}$ Divisão de Nefrologia da Escola de Ciências Médicas, UNICAMP - Campinas / SP

Correspondência:

Roberto C. Manfro

Serviço de Nefrologia. Unidade de Transplante Renal. Hospital de Clínicas de Porto Alegre Porto Alegre / RS - CEP: 90035-903 - Brasil

Fax: (51) 33598121

E-mail: rmanfro@hcpa.ufrgs.br
}

Recebido em: 14.05.2009

\section{INTRODUÇÃO}

A rejeição humoral aguda (RHA) é caracterizada por disfunção grave do enxerto, associada à presença de anticorpos doador-específicos. Geralmente ela é resistente ao tratamento com corticóides e terapia antilinfocítária e está associada a mau prognóstico do enxerto em receptores de transplante renal, com taxa de sobrevida do enxerto em um ano de 15 a 50\%. ${ }^{1}$ Os critérios diagnósticos para RHA incluem critérios morfológicos, imunopatológicos e sorológicos. O diagnóstico da RHA é então embasado na demonstração morfológica de injúria tecidual, depósitos de C4d em capilares peritubulares e evidências de atividade humoral específica contra o doador. ${ }^{2}$ Por estar fortemente associada à presença de anticorpos doador-específicos, a presença de depósitos da sub-fração C4d do complemento em capilares peritubulares apresenta alta sensibilidade e especificidade para o diagnóstico de RHA. ${ }^{3}$ Além disso, tal deposição tem forte correlação com má sobrevida dos enxertos. ${ }^{4}$ Anticorpos anti-HLA do doador têm sido identificados como causa primária da RHA. ${ }^{3}$

A remoção dos anticorpos anti-HLA é a meta primária do tratamento da RHA. A plasmaferese é um tratamento efetivo para tal remoção, mas leva à diminuição do catabolismo da IgG e o subseqüente aumento compensatório na síntese de anticorpos. ${ }^{5}$ Já as imunoglobulinas intravenosas (IVIg) em doses elevadas inibem a síntese de anticorpos ou produzem atividade anti-idiotípica através de mecanismos imunomodulatórios. ${ }^{6} \mathrm{~A}$ associação da plasmaferese com as IVIg tem sido descrita como efetiva no tratamento de RHA.,8 Neste estudo, relatamos a experiência inicial de quinze pacientes com RHA tratados com plasmaferese ou plasmaferese associada à IVIg, ATG ou OKT3. 


\section{PACIENTES E MÉTODOS}

Foi realizada uma análise retrospectiva de 507 receptores de transplantes de rim isolado entre janeiro de 2002 e abril de 2008. O diagnóstico de RHA foi baseado em critérios presentemente aceitos descritos acima. ${ }^{2}$ Quinze pacientes apresentaram diagnóstico de RHA e foram tratados com plasmaferese e imunoglobulinas intravenosas ou plasmaferese e ATG/OKT3.

Os dados analisados incluíram idade, sexo, cor, tipo de doador (vivo ou falecido), compatibilidade HLA, valor de reatividade contra painel de anticorpos (PRA) pré-transplante, prova cruzada póstransplante, presença de anticorpos doador-específicos, número do transplante, dia em que foi detectada a RHA, aspectos da biópsia renal, tratamento da RHA e evolução pós-tratamento.

Os dados são apresentados em números absolutos, porcentagens, médias e desvios-padrão.

O presente estudo foi aprovado pelo comitê de ética em pesquisa do Hospital de Clínicas de Porto Alegre.

\section{RESULTADOS}

Os principais resultados estão apresentados na Tabela 1.

Dos 507 transplantes de rim isolado realizados no período do estudo, foram identificados 15 casos de RHA, representando incidência de 2,95\%. Destes, a média de idade dos receptores foi de 41,86 $\pm 11,8$ anos (16 a 66 anos), 80\% do sexo feminino, 86,6\% caucasianos, $74 \%$ receptores de órgão de doador falecido e $20 \%$ retransplantes. Antes do transplante, todos os pacientes apresentaram prova cruzada por microlinfocitotoxicidade negativa para linfóticos T e B. Em quatro pacientes foi realizada citometria de fluxo para linfócitos T e B pré-transplante, todas negativas, exceto em um paciente que apresentou positividade fraca para linfócitos B. A média da reatividade contra painel foi de $59 \pm 42,8 \%$ (variação: 0 a $100 \%$ ). Oito pacientes receberam transfusões sangüíneas e oito apresentaram gestações previamente ao transplante. Sete pacientes (46,6\%) apresentaram reatividade contra painel $>20 \%$ e receberam indução com anticorpos, sendo quatro com Thymoglobulin ${ }^{\circledR}$ (Genzyme Corporation, Framingham, Massachusetts, USA) e três com Orthoclone-OKT3 ${ }^{\circledR}$ (OrthoBiotech Inc, Raritan, New Jersey, USA). Nos casos aqui descritos não foi realizada pesquisa de anti-MICA pré ou pós transplante. Os episódios de RHA foram detectados em média 15,5 $\pm 8,1$ dias após o transplante. No momento do diagnóstico da RHA, dez pacientes eram dependentes de diálise e a média da creatinina sanguínea foi de $5,1 \pm 2,5 \mathrm{mg} / \mathrm{dl}$. Os achados na biópsia renal foram: presença de neutrófilos nos capilares peritubulares $(80 \%)$, vasculite $(70 \%)$, necrose fibrinóide $(40 \%)$. Depósitos de C4d em capilares peritubulares foram encontrados nos onze casos em que a pesquisa foi feita (Figura 1).

Treze pacientes foram tratados com sessões de plasmaferese e em doze casos foi adicionado tratamento com IgIV, ATG ou OKT3. Dois pacientes foram tratados apenas com OKT3 ou ATG. O número de sessões de plasmaferese foi orientado pela resposta clínica, sendo

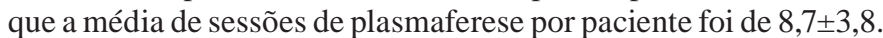
A média de infusões de IgIV foi $9,1 \pm 4,4$ doses por paciente.

Em um ano, a sobrevida dos pacientes foi de $100 \%(\mathrm{n}=15)$ e a dos enxertos de $73 \%(n=11)$. Entre pacientes que não perderam o enxerto, a creatinina média foi de $1,62 \pm 1,03 \mathrm{mg} / \mathrm{dL}$ no final do primeiro ano. Não houve associação da creatinina em um ano com o número de sessões de plasmaferese, dose de IgIV, número de transfusões prévias,
Tabela 1. Dados demográficos, esquemas terapêuticos e resposta ao tratamento em receptores de transplante renal com rejeição humoral aguda

\begin{tabular}{|c|c|}
\hline Variáveis e desfechos & Características e número de pacientes \\
\hline Media Idade do Receptor (amplitude) & 41,86 anos $(16-66)$ \\
\hline \multicolumn{2}{|l|}{ Raça } \\
\hline Caucasiano & 13 \\
\hline Afro-descendente & 2 \\
\hline $\operatorname{Sexo}(M / F)$ & $3 / 12$ \\
\hline Doador (Vivo/ Falecido) & $4 / 11$ \\
\hline \multicolumn{2}{|l|}{ Perfil Imunológico } \\
\hline Reatividade média contra painel & $59 \pm 42,8 \%$ \\
\hline Transfusões prévias & 8 pacientes \\
\hline Gestações prévias & 8 pacientes \\
\hline Re-transplantes & 3 pacientes \\
\hline \multicolumn{2}{|l|}{ Tratamento } \\
\hline$P F+I V I G$ & 7 pacientes $(46 \%)$ \\
\hline $\mathrm{PF}+\mathrm{ATG}$ & 4 pacientes $(26 \%)$ \\
\hline $\mathrm{PF}+0 \mathrm{KT} 3$ & 1 paciente $(7 \%)$ \\
\hline PF isolada & 1 paciente $(7 \%)$ \\
\hline ATG & 1 paciente $(7 \%)$ \\
\hline OKT3 & 1 paciente $(7 \%)$ \\
\hline \multicolumn{2}{|l|}{ Desfecho Clínico } \\
\hline Creatinina pré tto (média \pm DP) & $6,2 \mathrm{mg} / \mathrm{dl}(+3,8 \mathrm{mg} / \mathrm{dl})$ \\
\hline Creatinina pós tto (média \pm DP) & 3,7 mg/dl (+2,3 mg/dl) \\
\hline Creatinina em 1 ano (média \pm DP) & $1,4 \mathrm{mg} / \mathrm{dl}(+2,8 \mathrm{mg} / \mathrm{dl})$ \\
\hline Perda do enxerto & 4 pacientes $(27 \%)$ \\
\hline Sobrevida paciente em 1 ano & 15 pacientes $(100 \%)$ \\
\hline Sobrevida enxerto em 1 ano & 11 pacientes $(73 \%)$ \\
\hline
\end{tabular}

PF: plasmaferese, IVIG: imunoglobulinas intravenosas, ATG: Globulina anti timocitária, OKT3: muromonab, DP: desvio-padrao, M: masculino, F: feminino

Figura 1. Biópsia renal de paciente com rejeição humoral mostrando depósito difuso de C4D em capilares peritubulares.

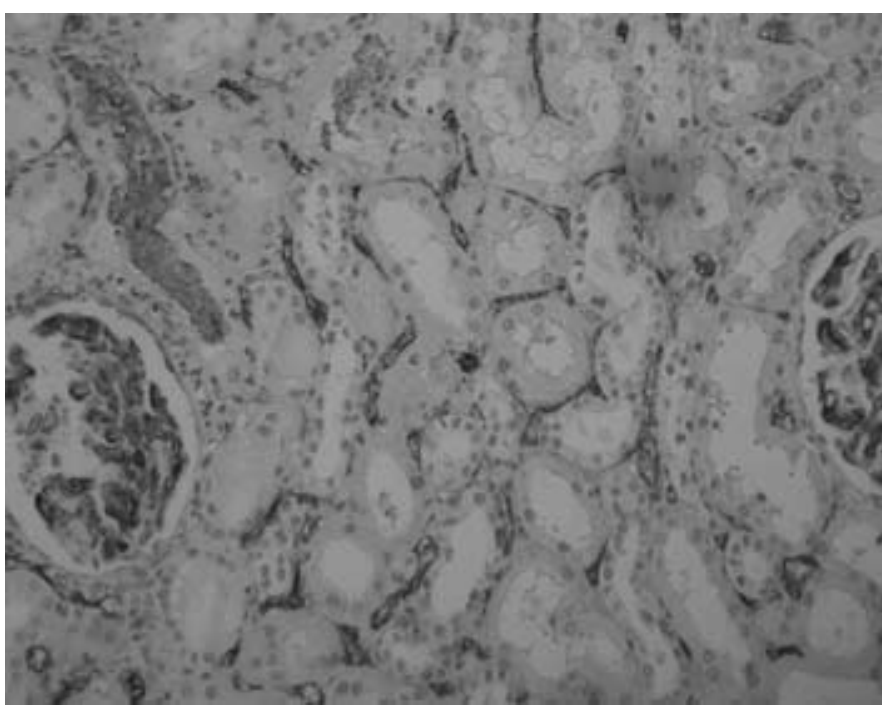


número de gestações prévias, creatinina no diagnóstico da RHA e creatinina no término do tratamento da RHA. Em um paciente ocorreu reversão completa do episódio de RHA com plasmaferese e IVIg, mas o mesmo desenvolveu sepse grave e a terapia imunossupressora foi descontinuada com subseqüente perda do enxerto renal.

Ao término do tratamento, três pacientes não recuperaram a função renal e permaneceram dependentes de diálise. Esses pacientes eram hipersensibilizados, com painel maior do que $75 \%$ e apresentaram disfunção inicial do enxerto, sendo o diagnóstico de rejeição humoral realizado através de biópsia de vigilância.

\section{DISCUSSÃO}

Por estar associada à elevada incidência de perda de enxerto, a rejeição humoral aguda (RHA), apesar de incomum, é uma condição grave em receptores de transplante renal, ocorrendo com maior freqüência em casos de elevado risco imunológico. Torna-se assim necessária a monitorização mais rigorosa desses pacientes, em especial daqueles com disfunção inicial dos enxertos (do inglês “delayed graft function”), quando a creatininemia não é um parâmetro utilizável para tal monitorização. Até recentemente, essa condição estava associada a prognóstico bastante sombrio. No entanto, o melhor entendimento de sua fisiopatologia levou à padronização diagnóstica e ao surgimento de novas abordagens terapêuticas com plasmaferese e imunoglobulinas intravenosas associadas à intensificação da imunossupressão, em geral com micofenolato mofetil/sódico e tacrolimus.

Nessa série, os 15 casos de RHA entre os 507 transplantes isolados de rim representaram incidência de $2,95 \%$ no período de sete anos, estando de acordo com os dados da literatura internacional, que variam de 3 a $10 \%{ }^{7,8,9}$ Neste estudo, caracteristicamente, a maioria dos pacientes apresentava sensibilização anti-HLA. No entanto, oito episódios ocorreram em pacientes não sensibilizados, o que demonstra o papel dos anticorpos anti-HLA de novo e, possivelmente, de anticorpos não dirigidos aos antígenos HLA, como os antiMICA, por exemplo. ${ }^{10}$ Todos os pacientes hipersensibilizados receberam indução com anticorpos anti-linfócitos T. Três pacientes que não apresentavam reatividade contra painel antes do transplante receberam pulsoterapia de corticosteróides e OKT3 previamente ao tratamento com plasmaferese e IgIV. Na presente casuística, três pacientes não responderam ao tratamento e permaneceram dependentes de diálise. Esses resultados são similares aos reportados por White et al e Ibernón et al, que utilizaram terapias semelhantes em seus relatos. ${ }^{8,11}$
Na presente série de casos, pudemos detectar duas características possivelmente associadas a não resposta ao tratamento empregado, quais sejam a intensidade da sensibilização para os antígenos HLA e a presença de disfunção inicial do enxerto. Nossos resultados corroboram os apresentados na literatura recente. White et al descreveram apenas uma perda de enxerto em uma série de nove casos de RHA tratados com plasmaferese associada à IGIV. ${ }^{8}$ Já Ibernón et al também demonstraram resultados semelhantes com esta abordagem em uma série de sete casos na qual a sobrevida do enxerto em um ano foi de $70 \%{ }^{11}$ Nessa última série de casos, um paciente recebeu também terapia com anticorpos anti-CD20 para recuperação da função renal.

Devido ao envolvimento de linfócitos B no processo de rejeição humoral, o uso de anticorpos anti-CD20 (Rituximab ${ }^{\circledR}$, Genentech Inc.) parece ser pertinente nessa condição. Em uma série de casos com oito pacientes com RHA tratados com Rituximab (3 a 5 doses semanais de $375 \mathrm{mg} / \mathrm{m}^{2}$ ) associados à plasmaferese, esteróides, micofenolato e tacrolimus, a sobrevida de pacientes e enxertos foi de $100 \%$ e $75 \%$, respectivamente, em seguimento de dez meses. ${ }^{12}$ Recentemente, este mesmo grupo publicou uma atualização de dados com uma série de 22 pacientes com rejeição humoral/vascular que receberam o mesmo tratamento acima descrito. Com um tempo de seguimento médio de nove meses (2-48) a sobrevida dos pacientes e do enxerto foi $86,4 \%$ e $77,3 \%$ respectivamente, mas foi associada a uma alta incidência de infecções graves. ${ }^{13}$ Estudo piloto com uma única dose fixa de Rituximab (500 mg) foi realizado em sete casos de pacientes que apresentaram rejeição mediada por anticorpos resistente à terapia padrão (plasmaferese e IVIg). O tempo de seguimento médio foi de 21 meses (9,5-33 meses) e a sobrevida do enxerto e do paciente foi de $100 \%$. Foi observada também baixa incidência de complicações infecciosas e neoplasias. ${ }^{14}$ Recentemente, foi também descrita a eficácia da droga Bortezomib (Velcade ${ }^{\circledR}$ Janssen-Cilag) em um número restrito de pacientes com rejeição mista (humoral e celular) refratárias às terapias acima descritas. ${ }^{15}$ Essas novas drogas são promissoras para o tratamento dessa situação e deverão ser testadas em ensaios clínicos controlados.

\section{CONCLUSÃO}

A plasmaferese em associação com IVIg, ATG ou OKT3 seguidos de terapia imunossupressora intensificada são eficientes no tratamento da rejeição humoral aguda, permitindo a sobrevida da maioria dos enxertos. Essa estratégia mostra-se promissora no tratamento de RHA, embora estudos adicionais randomizados e com maior número de pacientes sejam necessários para estabelecer a abordagem mais custo-efetiva nessa situação.

\section{ABSTRACT}

Introduction: Acute humoral rejection (AHR) is a severe complication for renal transplantation usually causing a poor graft prognosis. A recent therapeutic approach with plasmapheresis and intravenous immunoglobulins (IVIg) has being proposed. Purpose: To describe the effectiveness of the treatment of AHR. Patients and methods: From January 2002 to April 2008 fifteen patients were diagnosed as having AHR, and they were treated with plasmapheresis and IVIg or ATG/OKT3 in our institution. Results: Reversal of AHR was attained in twelve (80\%) patients. One-year patient and graft survivals were $100 \%$ and $73 \%$, respectively. One successfully treated patient had the immunosuppressive regimen discontinued due the presence of sepsis, and the graft was subsequently lost. Conclusion: We concluded that this is an efficient therapeutic, allowing the survival of the majority of grafts.

Keywords: Renal transplantation, Graft Rejection, Plasmapheresis, Intravenous Immunoglobulins, Antilynphocyte Serum, Muromonab-CD3. 


\section{REFERÊNCIAS}

1. Mauiyyedi S, Crespo M, Collins AB, Schneeberger EE, Pascual MA, Saidman SL, et al. Acute humoral rejection in Kidney transplantation: II. Morphology, immunopathology, and pathologic classification. J Am Soc Nephrol 2002;13:779-87.

2 Racusen LC, Halloran PF, Solez K. Banff 2003 meetinhg report: new diagnosis insights and standarts. Am J Transplant 2004;4:1562-6.

3. Koo DDH, Roberts ISD, Quiroga I, Procter J, Barnardo MCNM, Sutton M, et al. C4d deposition in early renal allograft protocol biopsies. Transplantation 2004;78:398-403.

4. Pascual M, Saidman S, Tolkoff-Rubin N, Williams WW, Mauiyyedi S, Duan JM, et al. Plama exchange and tacrolimus-mycophenolate rescue for acute humoral rejectin in kidney transplantation. Transplantation 1998;66:1460-64.

5. Csipo I, Kavai M, Kiss E, Bedo Z, Csongor J, Szegedi GY, et al. Serum complement activation of SLE patitents during plasmapheresis. Autoimmunity 1997:25:139-46.

6. Kazatchkine MD, Kaveri SV. Immunomodulation of autoimmune and inflammatory disease with intravenous immune globulin. N Eng J Med 2001;345:747-55.

7. Min L, Shuming J, Zheng T, Daxi J, Huiping C, Zhihong L, et al. Novel rescue therapy for C4D-positive acute humoral renalallograft rejection. Clin Tranplantation 2005; 19:51-5

8. White NB, Greenstein SM, Cantafio AW, Schechner R, Glicklich D, McDonough P, et al. Successful rescue therapy with plasmapheresis and intravenous immunoglobulin for acute humoral renal transplant rejection. Transplantation 2004:78:772-4.
9. Shah A, Nadasdy T, Arend L, Brennan J, Leong N, Coppage M, et al. Treatment of C4d-positive acute humoral rejection with plasmapheresis and rabbit polyclonal antithymocyte globulin. Transplantation 2004 May 15;77(9):1399-405.

10. Alvarez-Márquez A, Aguilera I, Gentil MA, Caro JL, Gernal G, Fernández AJ, et al. Donor-specific antibiodies against HLA, MICA, and GSTT1 in patientes with allograft rejection and C4D deposition in renal biopsies. Transplantation 2009;87:94-9.

11. Ibernón M, Gil-Vernet S, Carrera M, Serón D, Moreso F, Bestard O, et al. Therapy with plasmapheresis and intravenous immunoglobulin for acute humoral rejection in kidney transplantation. Transplant Proc 2005;37:3743-5.

12. Faguer S, Kamar N, Guilbeaud-Frugier C, Fort M, Modesto A, Arnaud M, et al. Rituximab therapy for acute humoral rejection after kidney transplantation. Transplantation 2007;83:1277-80.

13. Rostaing L, Guilbeaud-Frugier C, Kamar N. Rituximab for humoral rejection after kidney transplantantion: An update. Transplantation 2009;87:1261.

14. Mulley WR, Hudson F, Tait BD, Skene AM, Dowling JP, Kerr PG et al. A single low-fixed dose of rituximab to salvage renal transplants from refractory antibodymediated rejection. Transplantation 2009;87:286-9.

15. Everly MJ, Everly JJ, Susskind B, Brailey P, Arend LJ, Alloway RR, et al. Bortezomib provides effective therapy for antibody- and cell-mediated acute rejection. Transplantation 2008;86:1754-61. 